B. Bellaccini

Nagoya Math. J.

Vol. 89 (1983), 109-118

\title{
PROPER MORPHISMS AND EXCELLENT SCHEMES
}

\author{
BARBARA BELLACCINI
}

\section{Introduction}

Let $f: X \rightarrow Y$ be a finite type morphism of locally noetherian schemes. It is well known $([3, I V, 7.8 .6])$ that the excellent property ascends from $Y$ to $X$. On the other side there are counter-examples where $X$ is excellent and $Y$ is not. First of all it is easy to show that the condition on chains of prime ideals does not descend (see [3, IV, 7.8.4]), even by finite morphisms. Secondly in [2] it is produced an example where $X$ is excellent while $Y$ is not a $G$-scheme (i.e. it has not the good properties of formal fibers). However in [2] it is also proved that the property concerning the openness of regular loci (the so called " $J-2$ ") descends by finite type surjective morphisms. Therefore we are led to the following question: When does the $G$-scheme property descend? I.e. what conditions do we need on $f$ ? A reasonable condition is conjectured (in [2]) as the following: $f$ is proper surjective. The aim of the present paper is precisely to give an answer to such a question. What we really prove is the following. If $X$ is a $G$-scheme and $J$-2 (quasi-excellent), then the same is true for $Y$, provided that $f$ is proper surjective and moreover all the residue fields of $Y$ have characteristic 0 . We remark that the result is strongly based on Hironaka's desingularization for quasi-excellent schemes defined over a field of characteristic 0 .

I wish to thank Prof. Paolo Valabrega for several useful conversations on the subject of this paper.

\section{$\S 0$. Recalls and definitions}

All rings are assumed to be commutative noetherian rings with unit and all schemes are assumed to be locally noetherian.

1. We recall that a graded ring is a ring $S$ with a direct decompo-

Received June 15, 1981. 
sition of the underlying additive group, $S=\oplus_{n=0}^{\infty} S_{n}$, such that $S_{n} S_{m} \subseteq S_{n+m}$ for every $n, m \geqslant 0$.

An element of $S_{n}$ is called a homogeneous element of degree $n$.

$S_{0}$ is a subring of $S$ and $S_{+}=\oplus_{n>0} S_{n}$ is an ideal of $S$.

An ideal $\mathfrak{J}$ of $S$ is homogeneous if it has a basis consisting of homogeneous elements.

A homogeneous ideal $\mathfrak{\Im}$ of $S$ is irrelevant if $\sqrt{\mathfrak{S}} \supseteq S_{+}$and otherwise it is relevant.

Since $S$ is noetherian, $S$ is finitely generated as an $S_{0}$-algebra.

Convention: Once for all we assume that the graded $S_{0}$-algebra $S=$ $\oplus_{n=0}^{\infty} S_{n}$ is generated over $S_{0}$ by $x_{0}, \cdots, x_{n} \in S_{1}$, say $S=S_{0}\left[x_{0}, \cdots, x_{n}\right]$.

2. Let $\operatorname{Proj}(S)=\{\mathfrak{\beta} \in \operatorname{Spec}(S) / \mathfrak{R}$ is a homogeneous relevant ideal $\}$. We can give Proj $(S)$ a structure of scheme. For this construction and for the properties of Proj $(S)$ we refer to [4]. (See also [3, II] where homogeneous prime ideals are defined in a slightly different but equivalent way).

3. The dimension of a scheme $X$, denoted by $\operatorname{dim}(X)$, is its dimension as a topological space. If $X=\operatorname{Spec}(A)$ for a $\operatorname{ring} A$, then the dimension of $X$ is the same as the Krull dimension of $A$ and we shall write as $\operatorname{dim}(A)$. If $X=\operatorname{Proj}(S)$ then $\operatorname{dim}(X)=d$ means that there exists a chain $\mathfrak{p}_{0} \subsetneq \mathfrak{p}_{1} \subsetneq \cdots \subsetneq \mathfrak{p}_{r}$ of relevant homogeneous prime ideals in $S$, while no such chain of length $r+1$ exists (see [3, II, 2.3.17]).

4. Let $X$ be an integral scheme. We denote by $\phi(X)$ the function field of $X$. For a ring $A$ we shall write $\phi(A)$ instead of $\phi(\operatorname{Spec}(A))$.

5. A ring $A$ is quasi-excellent (QE for short) iff:

(i) $A$ is a $G$-ring, i.e. the formal fibers of $A$ are geometrically regular.

(ii) $A$ is $J-2$; i.e. the regular locus of $\operatorname{Spec}\left(A^{\prime}\right)$ is Zariski open whenever $A^{\prime}$ is any $A$-algebra of finite type.

A ring $A$ is excellent if it is QE and universally catenary (UC for short).

A scheme $X$ is excellent (resp. QE) if there exists a covering of $X$ by open affine subsets $U_{i}=\operatorname{Spec}\left(A_{i}\right)$ such that $A_{i}$ is excellent (resp. QE), for each $i$.

For excellent rings and schemes we refer to [3, $\left.\mathrm{IV}_{2}\right]$ and [6, chap. 13].

6. Let $f: X \rightarrow Y$ be a scheme morphism. $f$ is proper if it is separated, of finite type and universally closed. $f$ is projective if it factors into a 
closed immersion $i: X \rightarrow P_{Y}^{n}$ for some $n$, followed by the projection $P_{Y}^{n} \rightarrow$ $Y\left(P_{Y}^{n}\right.$ denotes the projective $n$-space over $\left.Y\right)$.

Example. Let $A$ be a ring, let $S$ be a graded ring with $S_{0}=A$, which is finitely generated by the elements of $S_{1}$ as an $S_{0}$-algebra. Then the natural map $\operatorname{Proj}(S) \rightarrow \operatorname{Spec}(A)$ is a projective morphism.

7. Remark. Let $A$ be a ring. A scheme $Y$ over $\operatorname{Spec}(A)$ is projective if and only if it is isomorphic to $\operatorname{Proj}(S)$ for some graded ring $S$, where $S_{0}=A$, and $S$ is finitely generated by the elements of $S_{1}$ as an $S_{0}$-algebra ([4, II, 5.18]).

8. Let $X, Y$ be two reduced schemes. A morphism $f: X \rightarrow Y$ is birational if for every maximal point $y \in Y, f^{-1}\{y\}=\{x\}$ with $x$ maximal point of $X$ and if the homomorphism between the residue fields $k(y) \rightarrow k(x)$ deduced by $f$ is a bijection. ([3, IV, 6.15.4]).

If both $X$ and $Y$ are integral schemes, then the generic points of $X$ and $Y$ correspond through $f$ and the fraction fields of $X$ and $Y$ are isomorphic.

9. Let $X$ be a reduced scheme. A scheme $Y$ is a resolution of singularities of $X$ if there is a proper and birational morphism $f: Y \rightarrow X$ and $Y$ is regular. If such $Y$ exists, then we say that $X$ is desingularizable.

10. We recall the following results on resolution of singularities due to Hironaka ((a)) and Grothendieck ((b), (c)):

(a) Let $X$ be a reduced noetherian scheme with all the residue fields of characteristic 0 . If $X$ is $\mathrm{QE}$ then $X$ is desingularizable.

(b) Let $X$ be a locally noetherian scheme. If any integral finite $X$-scheme is desingularizable, then $X$ is $\mathrm{QE}$.

(c) Let $X$ be a locally noetherian scheme such that all the residue fields of $X$ have characteristic 0 . If every closed integral subscheme of $X$ is desingularizable, then $X$ is $\mathrm{QE}$.

For more details see [5], [3, IV, 7.9.5] and also [7, Proposition 3.1., Example 3 and Theorem 3.2 with Remark 1].

$\S 1$.

The present section is concerned with some preliminary results on the graded $S_{0}$-algebra $S$ and on Proj $(S)$. Mainly we will see when $\operatorname{Proj}(S)$ and $\operatorname{Spec}\left(S_{0}\right)$ have the same dimension and when $\phi(\operatorname{Proj}(S))$ is a finite algebraic extension of $\phi\left(S_{0}\right)$. 
Lemma 1.1. Let $S_{0}$ be a domain and $S=S_{0}\left[x_{0}, \cdots, x_{n}\right]$ a graded $S_{0^{-}}$ algebra generated by $x_{0}, \cdots, x_{n} \in S_{1} . \quad$ Let $f: \operatorname{Proj}(S) \rightarrow \operatorname{Spec}\left(S_{0}\right)$ be a scheme morphism. Consider the following conditions:

(a) For each $i(0 \leqslant i \leqslant n)$ and for each $\mathfrak{P} \in \operatorname{Proj}(S)$ with $x_{i} S \subseteq \mathfrak{P}$, it holds $\mathfrak{B} \cap S_{0} \neq(0)$.

(b) For each $i, S_{+}$is a minimal prime ideal of $x_{i} S$.

(c) There exists $i$ such that $S_{+}$is a minimal prime ideal of $x_{i} S$.

Then we have: $(\mathrm{a}) \rightarrow(\mathrm{b}) \rightarrow(\mathrm{c})$.

Proof. (a) $\rightarrow$ (b). By (a) it follows that there is an irrelevant ideal $\mathfrak{Q} \in \operatorname{Ass}\left(x_{i} S\right)$ such that $\mathfrak{Q} \cap S_{0}=(0)$. In fact assume the contrary and consider $\sqrt{x_{i} S}=\bigcap_{j=1}^{k} \mathfrak{Q}_{j}$ where $\mathfrak{Q}_{j} \in \operatorname{Ass}\left(x_{i} S\right)$ for $1 \leqslant j \leqslant k$. Then $\mathfrak{Q}_{j} \cap$ $S_{0} \neq(0)$ for each $j$ and $\sqrt{x_{i} S} \cap S_{0} \neq(0)$. But this means that there are $t \in S_{0}, t \neq 0$ and $r \in N$ such that $t^{r} \in x_{i} S$, and this is absurd because the degree of $t^{r}$ is zero if $t \in S_{0}$ while the elements of $x_{i} S$ have positive degree.

So there exists an irrelevant minimal prime ideal $\mathfrak{Q}$ of $x_{i} S$ with $\mathfrak{\cap}$ $S_{0}=(0)$. But $\mathfrak{\supseteq} S_{+}$because it is irrelevant and $\mathfrak{\subseteq} S_{+}$because $\mathfrak{Q} \cap S_{0}$ $=(0)$. Therefore $\mathfrak{Q}=S_{+}$.

(b) $\rightarrow$ (c). Obvious.

LEMMA 1.2. Let $S$ be a graded ring, with $S_{0}$ domain, and assume that $f: \operatorname{Proj}(S) \rightarrow \operatorname{Spec}\left(S_{0}\right)$ is a surjective morphism. Then there exists a homogeneous relevant prime ideal $\mathfrak{P}_{0}$ of $S_{0}$ such that the induced morphism $f^{\prime}: \operatorname{Proj}\left(S / P_{0}\right) \rightarrow \operatorname{Spec}\left(S_{0}\right)$ is again surjective.

Proof. Since $(0) \in \operatorname{Spec}\left(S_{0}\right)$ and $f$ is surjective, there exists $\mathfrak{P}_{0} \in \operatorname{Proj}(S)$ such that $\mathfrak{P}_{0} \cap S_{0}=(0)$. Now consider the following diagram

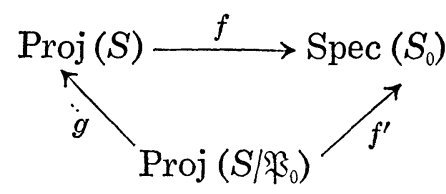

where $g$ is the closed immersion determined by the surjective homomorphism of graded rings $S \rightarrow S / \mathfrak{P}_{0}$ and $f^{\prime}=f \circ g$. Then $f^{\prime}$ is surjective because it is proper, hence closed and $(0) \in \operatorname{Im}\left(f^{\prime}\right)$.

Proposition 1.3. Let $S_{0}$ be a domain and let $S=S_{0}\left[x_{0}, \cdots, x_{n}\right]$ be a graded domain generated by $x_{0}, \cdots, x_{n} \in S_{1}$ over $S_{0}$. Let $f: \operatorname{Proj}(S) \rightarrow$ $\operatorname{Spec}\left(S_{0}\right)$ be a surjective morphism. Consider the following conditions:

(d) $\operatorname{dim}\left(S_{0}\right)=\operatorname{dim}(\operatorname{Proj}(S))$. 
(e) $\phi(\operatorname{Proj}(S))$ is a finite algebraic extension of $\phi\left(S_{0}\right)$. Then condition (c) of 1.1 implies (d) and (d) implies (e).

Proof. $(\mathrm{c}) \rightarrow(\mathrm{d})$. The morphism $f: \operatorname{Proj}(S) \rightarrow \operatorname{Spec}\left(S_{0}\right)$ is closed and surjective so $\operatorname{dim}(\operatorname{Proj}(S)) \geqslant \operatorname{dim}\left(S_{0}\right)$ ([3, IV, 5.4 .1 (ii)]). Now we distinguish two cases:

(i) $\operatorname{dim}\left(S_{0}\right)=+\infty$. Then, by the foregoing inequality, $\operatorname{dim}(\operatorname{Proj}(S))$ $=+\infty$, that is (d) holds.

(ii) $\operatorname{dim}\left(S_{0}\right) \supsetneqq+\infty$. Then it is enough to verify the inequality $\operatorname{dim}(\operatorname{Proj}(S)) \leqslant \operatorname{dim}\left(S_{0}\right)$. It is clear that (ii) implies $\operatorname{dim}(S) \supsetneqq+\infty$.

Let $\mathfrak{Q}=\left(x_{0}, \cdots, x_{n}\right)$, then ht $(\mathfrak{Q}) \leqslant 1$ by (c). But $\mathfrak{Q} \neq(0)$ implies ht $(\mathfrak{Q})=1$ by the hypothesis that $S$ is a domain.

Now we prove that $\operatorname{dim}(S)=\operatorname{dim}\left(S_{0}\right)+1$. We have $\operatorname{dim}(S)-$

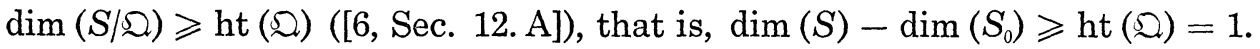
On the other hand, we compute the dimension of the fiber of the natural morphism $\varphi: \operatorname{Spec}(S) \rightarrow \operatorname{Spec}\left(S_{0}\right)$ over the generic point $(0) \in \operatorname{Spec}\left(S_{0}\right)$, i.e. $\operatorname{dim}\left(\phi\left(S_{0}\right)\left[x_{0}, \cdots, x_{n}\right]\right)=\operatorname{dim}\left(\phi\left(S_{0}\right)\left[x_{0}, \cdots, x_{n}\right] /\left(x_{0}, \cdots, x_{n}\right)\right)+\operatorname{ht}\left(x_{0}, \cdots, x_{n}\right)$ $=1\left(\left[6\right.\right.$, Sec. 14.H]). Since we have $\operatorname{dim}(S)-\operatorname{dim}\left(S_{0}\right) \leqslant \operatorname{dim}\left(\phi\left(S_{0}\right)\left[x_{0}, \cdots\right.\right.$, $\left.\left.x_{n}\right]\right)\left(\left[4\right.\right.$, II, Example 3.22]) we get $\operatorname{dim}(S) \leqslant \operatorname{dim}\left(S_{0}\right)+1$, hence $\operatorname{dim}(S)=$ $\operatorname{dim}\left(S_{0}\right)+1$.

On the other hand, since Proj $(S)$ is a topological subspace of Spec $(S)$, it is true that $\operatorname{dim}(\operatorname{Proj}(S)) \leqslant \operatorname{dim}(S)$. If we show that $\operatorname{dim}(S) \supsetneqq$ $\operatorname{dim}(\operatorname{Proj}(S))$, then by the foregoing inequality, we may deduce $\operatorname{dim}(\operatorname{Proj}(S))$ $=\operatorname{dim}\left(S_{0}\right)$.

Now, let $\mathfrak{q}_{0} \subsetneq \cdots \subsetneq \mathfrak{q}_{r}$ be a maximal chain of homogeneous primes of $\operatorname{Proj}(S)$ such that $\operatorname{dim}(\operatorname{Proj}(S))=r$. Consider the ideal $\mathfrak{q}^{\prime}$ of $S$ generated by $\mathfrak{q}_{r}$ and $x_{0}, \cdots, x_{n}$. Then $\mathfrak{q}^{\prime}$ is proper and different from $\mathfrak{q}_{r}$, because otherwise $x_{0}=\cdots=x_{n}=0$, but in this case $\operatorname{Proj}(S)=\emptyset$ and so $\operatorname{dim}(\operatorname{Proj}(S)) \leqslant \operatorname{dim}\left(S_{0}\right)$. Let $\mathfrak{P}$ be a minimal prime ideal of $\mathfrak{q}^{\prime}$. Then $\mathfrak{q}_{0} \subseteq \cdots \subseteq \mathfrak{q}_{r} \subseteq \mathfrak{P}$ is a chain of $\operatorname{Spec}(S)$, that is $\operatorname{dim}(S) \geqslant r+1>$ $\operatorname{dim}(\operatorname{Proj}(S))$.

$(\mathrm{d}) \rightarrow(\mathrm{e})$. Since $f: \operatorname{Proj}(S) \rightarrow \operatorname{Spec}\left(S_{0}\right)$ is a surjective morphism of integral schemes of the same dimension by (d), the fiber over the generic point of Spec $\left(S_{0}\right)$ has dimension 0 and hence it is finite. By [4, II, Example 3.7], it follows that $\phi(\operatorname{Proj}(S))$ is a finite field extension of $\phi\left(S_{0}\right)$.

Proposition 1.4. Let $S_{0}$ be a domain and let $S=S_{0}\left[x_{0}, \cdots, x_{n}\right]$ be a graded domain generated by $x_{0}, \cdots, x_{n} \in S_{1}$ over $S_{0}$. Let $f: \operatorname{Proj}(S) \rightarrow$ 
Spec $\left(S_{0}\right)$ be a surjective morphism. If the condition (e) of 1.3 holds then there exist a finite extension $S_{0}^{\prime}$ of $S_{0}$ and a proper birational morphism $g$ : $\operatorname{Proj}(S) \rightarrow \operatorname{Spec}\left(S_{0}^{\prime}\right)$ such that the following diagram

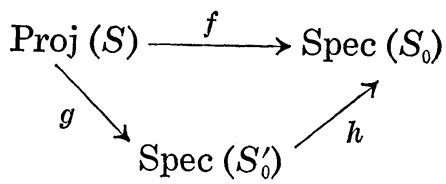

is commutative.

Proof. Observe that, if we define a finite extension $S_{0}^{\prime}$ of $S_{0}$ such that there exists a morphism $g: \operatorname{Proj}(S) \rightarrow \operatorname{Spec}\left(S_{0}^{\prime}\right)$ which makes the diagram commutative, then we deduce that $g$ is proper. In fact, since $f(=h \circ g)$ and $h$ are proper (in addition $h$ is finite), $g$ is also such ([4, II, 4.8. (e)]).

Now we have to define $S_{0}^{\prime}$ such that $g$ is birational too. Consider the integral closure $X^{\prime \prime}$ of $\operatorname{Spec}\left(S_{0}\right)$ in $\operatorname{Proj}(S)$ ([3, II, 6.3]). Then $X^{\prime \prime}$ is an affine scheme $\operatorname{Spec}\left(S_{0}^{\prime \prime}\right)$, because the morphism $h^{\prime}: X^{\prime \prime} \rightarrow \operatorname{Spec}\left(S_{0}\right)$ is integral. Moreover there is a natural morphism $g^{\prime}: \operatorname{Proj}(S) \rightarrow X^{\prime \prime} . \quad S_{0}^{\prime}$ is a suitable subring of $S_{0}^{\prime \prime}$. In fact, let $L=\phi(\operatorname{Proj}(S))$ and $K=\phi\left(S_{0}\right)$, then by (e) it follows that $L=K\left[t_{1}, \cdots, t_{m}\right]$, where $t_{i}$ is algebraic over $K$ for $i=1, \cdots, m$. Let $f_{i}(X)$ be the minimal polynomial of $t_{i}$ over $K(1 \leqslant i \leqslant$ $m)$, then it holds $f_{i}\left(t_{i}\right)=t_{i}^{s_{i}}+\left(a_{i 1} / b_{i 1}\right) t_{i}^{s_{i}-1}+\cdots+\left(a_{i s_{i}} / b_{i s_{i}}\right)=0$ where $a_{i j}$, $b_{i j} \in S_{0}$ for $1 \leqslant j \leqslant s_{i}$. Multiplying this equation by $\left(b_{i 1} \cdots b_{i s_{i}}\right)^{s_{i}}=b_{i}^{s_{i}}$, it becomes an equation of integral dependence for $b_{i} t_{i}$ over $S_{0}$. Put $S_{0}^{\prime}=$ $S_{0}\left[b_{1} t_{1}, \cdots, b_{m} t_{m}\right]$. Then $S_{0}^{\prime}$ is finite as an $S_{0}$-module and clearly $\phi\left(S_{0}^{\prime}\right)=$ $\phi(\operatorname{Proj}(S))$. Moreover there is a morphism $g^{\prime \prime}: \operatorname{Spec}\left(S_{0}^{\prime \prime}\right) \rightarrow \operatorname{Spec}\left(S_{0}^{\prime}\right)$. If we put $g=g^{\prime \prime} \circ g^{\prime}$, then $g$ is a proper and birational morphism.

$\S 2$.

Here we prove our main theorem on descent of excellent property by proper surjective morphisms.

THEOREM 2.1. Let $Y$ be a locally noetherian scheme defined over a field of characteristic 0 . Suppose $f: X \rightarrow Y$ is a proper surjective scheme morphism, then $X$ is $Q E$ if and only if $Y$ is $Q E$.

Proof. The "if" part is clear by definition (see [6 chap. 13]). Conversely, in our hypothesis we may apply 2.3 of [2] and we deduce that $Y$ is $J$-2. So it is enough to show that $Y$ is a $G$-scheme.

We verify that we may assume: 
1) $Y$ is affine, say $Y=\operatorname{Spec}\left(S_{0}\right)$.

2) $S_{0}$ in 1) is a domain.

3) $S_{0}$ is local.

1) In fact, if $\left\{V_{i}\right\}$ is an open affine covering of $Y$ and $U_{i}=f^{-1}\left(V_{i}\right)$, then $f_{\mid U_{i}}: U_{i} \rightarrow V_{i}$ is proper ([4, II, 4.8]) and surjective (since $f$ is surjective it follows $\left.f\left(f^{-1}\left(U_{i}\right)\right)=V_{i}\right) . \quad V_{i}$ satisfies the hypotheses and $U_{i}$ is QE for any $i$. Hence it suffices to prove that $V_{i}$ is $Q E$, but this means that we may assume $Y=\operatorname{Spec}\left(S_{0}\right)$.

2) It is known that $Y=\operatorname{Spec}\left(S_{0}\right)$ is a $G$-scheme if and only if $\operatorname{Spec}\left(S_{0} / \Re\right)$ is a $G$-scheme for every $\mathfrak{R} \in \operatorname{Spec}\left(S_{0}\right)$ with ht $(\mathfrak{P})=0$.

Let $\mathfrak{B} \in \operatorname{Spec}\left(S_{0}\right)$ with ht $(\mathfrak{P})=0$. For proper and surjective morphism $f: X \rightarrow \operatorname{Spec}\left(S_{0}\right)$, let $f^{\prime}$ be the morphism obtained from $f$ by the base extension $h: \operatorname{Spec}\left(S_{0} / \mathfrak{R}\right) \rightarrow \operatorname{Spec}\left(S_{0}\right)$, where $h$ is finite. Now consider the following diagram

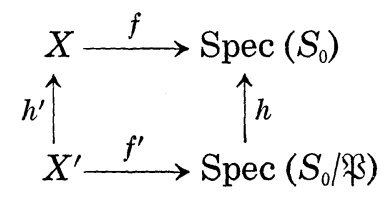

where $X^{\prime}=X \otimes_{\text {Spee }\left(S_{0}\right)} \operatorname{Spec}\left(S_{0} / \Re\right)$. Then $f^{\prime}$ is proper and surjective (such properties are stable under base extension by [3, II, 5.4.2 and I, 3.5.2]) and $X^{\prime}$ is QE because $h^{\prime}$, obtained from $h$ by the base extension $f$, is finite and $X$ is $\mathrm{QE}$ by hypothesis. Hence it follows that we may assume $S_{0}$ is a domain.

3) Proceeding similarly to point 2)-that is using the fact that our properties are stable under base extension-we show that $S_{0}$ may be taken local.

It is known that $S_{0}$ is a $G$-ring if and only if $\left(S_{0}\right)_{m}$ is a $G$-ring for every $m \in \operatorname{Max}\left(S_{0}\right)$. For $f: X \rightarrow Y=\operatorname{Spec}\left(S_{0}\right)$, let $f^{\prime}$ be the morphism obtained from $f$ by the base extension $h$ : Spec $\left(\left(S_{0}\right)_{\mathfrak{m}}\right) \rightarrow \operatorname{Spec}\left(S_{0}\right)$, where $h$ is a morphism essentially of finite type. We have

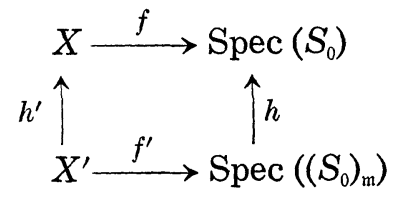

where $X^{\prime}=X \otimes_{\operatorname{Spec}\left(S_{0}\right)} \operatorname{Spec}\left(\left(S_{0}\right)_{\mathrm{m}}\right)$. Then $f^{\prime}$ is proper and surjective and $X^{\prime}$ is $\mathrm{QE}$ because $h^{\prime}$ is essentially of finite type. So we may assume that $S_{0}$ is local. 
Summarizing, we have a proper and surjective morphism $f: X \rightarrow Y$ where $X$ is $\mathrm{QE}$ and $Y=\operatorname{Spec}\left(S_{0}\right)$ with $S_{0}$ a local domain. In this case we may apply 5.6 .2 of $[3, \mathrm{II}]$ and we find a projective scheme $X^{\prime}$ over $\operatorname{Spec}\left(S_{0}\right)$ and a morphism $g: X^{\prime} \rightarrow X$ projective and surjective. The scheme $X^{\prime}$ is isomorphic to Proj $(S)$ for some graded ring $S$ (Remark 7). Then $\operatorname{Proj}(S)$ is QE because this property ascends by $g$, and $h=f \circ g: X^{\prime} \rightarrow Y$ is surjective because it is the composition of two surjective morphisms.

By 1.2 we may replace $S$ by $S / \Re_{0}$ for a suitable $\mathfrak{P}_{0} \in \operatorname{Proj}(S)$ and assume that $S$ is a domain. Now it is enough to show the theorem with $X=$ $\operatorname{Proj}(S)$ and $Y=\operatorname{Spec}\left(S_{0}\right)$, where $S_{0}$ is a local domain and $S=S_{0}\left[x_{0}, \cdots\right.$, $\left.x_{n}\right]$ is a domain.

We proceed by double induction with respect to $(n, d)$ where $n$ is the number of minimal generators of $S$ over $S_{0}$ and $d=\operatorname{dim}\left(S_{0}\right)$. Note that assuming $S_{0}$ local it holds $\operatorname{dim}\left(S_{0}\right) \supsetneqq+\infty$ and so the proof by induction covers all the cases.

For $(0, d)$ it holds $S=S_{0}\left[x_{0}\right]$. If we prove that $x_{0}$ is transcendental over $S_{0}$, then we see that $S$ is isomorphic to $S_{0}\left[X_{0}\right]$ with $X_{0}$ indeterminate and $\operatorname{Spec}\left(S_{0}\right)$ is isomorphic to $\operatorname{Proj}(S)$.

We show by absurdity that $x_{0}$ is transcendental over $S_{0}$. So suppose that we have an equation $a_{0}+a_{1} x_{0}+a_{2} x_{0}^{2}+\cdots+a_{m} x_{0}^{m}=0$ of algebraic dependence for $x_{0}$ of minimal degree $m$ with $a_{i} \in S_{0}$. Then $a_{0} \in S_{0} \cap\left(x_{0}\right)=$ $(0)$, i.e. $a_{0}=0$. But this means that $x_{0}$ is a zero-divisor, and this is impossible because $S$ is a domain.

For $(n, 0)$, it follows immediately that $S_{0}$ is $\mathrm{QE}$. In fact it is a field.

Assuming that the theorem is true for $(n-1, d)$ and $(n, d-1)$, we prove it for $(n, d)$. We distinguish two cases:

Case 1. There exist $i(0 \leqslant i \leqslant n)$ and $\mathfrak{P} \in \operatorname{Proj}(S)$ with $x_{i} S \subseteq \mathfrak{P}$ such that $\mathfrak{\beta} \cap S_{0}=(0)$. Take such a $\Re \in \operatorname{Proj}(S)$ and consider the quotient $S / \Re$. The surjective homomorphism of graded rings $S \rightarrow S / \mathfrak{R}$ gives rise to a closed immersion $g: \operatorname{Proj}(S / \mathfrak{P}) \rightarrow \operatorname{Proj}(S)$ which, in particular, is of finite type, hence the QE property ascends to Proj $(S / \mathfrak{P})$ ) from Proj $(S)$. Consider the following commutative diagram

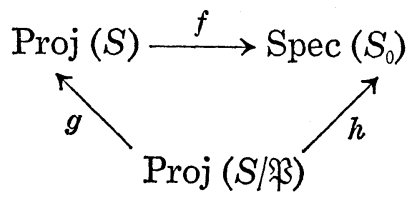


Obviously $h$ is projective ([4, II, Example 4.8.1]). Moreover, in our case, the prime ideal (0) of $S_{0}$ belongs to $\operatorname{Im}(h)$ and, since $h$ is closed, we have $(\overline{0}) \subseteq \operatorname{Im}(h)$, that is $h$ is surjective.

Applying now the inductive hypothesis we get that $S_{0}$ is QE. (In fact $x_{i} \in \mathfrak{P}$ hence the number of generators of $S / \mathfrak{P}$ over $S_{0}$ is strictly less than $n$.)

Case 2. For each $i(0 \leqslant i \leqslant n)$ and for each $\mathfrak{B} \in \operatorname{Proj}(S)$ with $x_{i} S \subseteq \mathfrak{P}$, it holds that $\mathfrak{R} \cap S_{0} \neq(0)$. In that case condition (a) of 1.1 holds. Then applying 1.1, 1.3, and 1.4, we have a ring $S_{0}^{\prime}$ finite over $S_{0}$ and a proper birational morphism $g: \operatorname{Proj}(S) \rightarrow \operatorname{Spec}\left(S_{0}^{\prime}\right)$. Moreover the morphism $h$ : $\operatorname{Spec}\left(S_{0}^{\prime}\right) \rightarrow \operatorname{Spec}\left(S_{0}\right)$ defined in 1.4 is finite and surjective. Hence by [2, 1.3], it suffices to verify that $S_{0}^{\prime}$ is a $G$-ring. We recall that $S_{0}$ and $S_{0}^{\prime}$ have the residue fields of characteristic 0 . So, in order to see that $S_{0}^{\prime}$ is a $G$-ring, it is sufficient to verify that every closed integral subscheme of $\operatorname{Spec}\left(S_{0}^{\prime}\right)$ is desingularizable. (See $0.10(\mathrm{c})$ ).

First prove that $S_{0}^{\prime}$ is desingularizable. In fact $\operatorname{Proj}(S)$ satisfies the hypothesis of Hironaka's theorem $(0.10(\mathrm{a}))$ and it is desingularizable. Let $Z$ be a resolution of Proj $(S)$, then, through the morphism $g:$ Proj $(S) \rightarrow$ $\operatorname{Spec}\left(S_{0}^{\prime}\right), Z$ resolves also $\operatorname{Spec}\left(S_{0}^{\prime}\right)$.

Now we see that every integral quotient $S_{0}^{\prime} / \Re$ is desingularizable. For $\mathfrak{B} \in \operatorname{Spec}\left(S_{0}^{\prime}\right)$, put $\mathfrak{p}=\mathfrak{B} \cap S_{0}$. Then $\mathfrak{P} \neq(0)$ implies $\mathfrak{p}=\mathfrak{R} \cap S_{0} \neq(0)$ because $S_{0}^{\prime}$ is integral over $S_{0}$. For the morphism $f: \operatorname{Proj}(S) \rightarrow \operatorname{Spec}\left(S_{0}\right)$ take the base extension $\varphi: \operatorname{Spec}\left(S_{0} / \mathfrak{p}\right) \rightarrow \operatorname{Spec}\left(S_{0}\right)$ and consider the following diagram

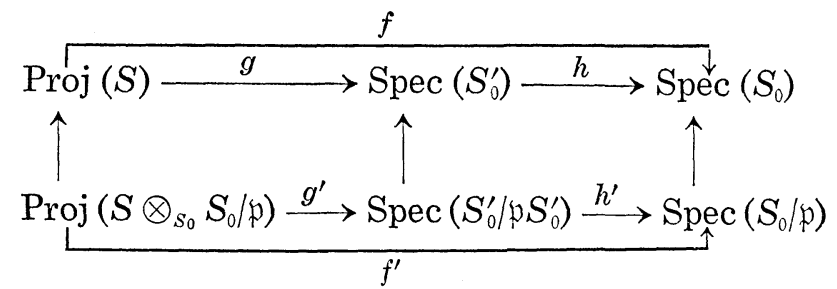

where the morphism $f^{\prime}$ and $h^{\prime}$ are obtained by $\varphi$ respectively from $f$ and $h$. Then $f^{\prime}$ is a surjective and projective morphism by [3, II, 5.6.5. and I, 3.5.2.] and Proj $\left(S \otimes_{S_{0}} S_{0} / \mathfrak{p}\right)$ is clearly $Q E$. Since $\mathfrak{p} \neq(0)$, we have $\operatorname{dim}\left(S_{1} / \mathfrak{p}\right)$ $§ \operatorname{dim}\left(S_{0}\right)$ and applying the inductive hypothesis, we deduce that $S_{0} / \mathfrak{p}$ is QE. Moreover since $h^{\prime}$ is finite ([3, II, 6.1.5]) the QE property passes from $S_{0} / \mathfrak{p}$ to $S_{0}^{\prime} / \mathfrak{p} S_{0}^{\prime}$ and from $S_{0}^{\prime} / \mathfrak{p} S_{0}^{\prime}$ to the quotient $S_{0}^{\prime} / \mathfrak{P}\left(\mathfrak{P} \supseteq \mathfrak{p} S_{0}^{\prime}\right)$. Therefore, by Hironaka's theorem, $S_{0}^{\prime} / \Re$ is desingularizable. This concludes our 
proof: We have seen that $S_{0}^{\prime}$ is a $G$-ring, so also $S_{0}$ is a $G$-ring, hence QE.

Remark 2.2. We need in our proof of the fact that Proj $(S)$ is desingularizable. Therefore we use both the $G$-scheme and the $J$-2 properties. We are not able to make the $G$-scheme property descend separately.

Remark 2.3. The UC property does not descend by proper surjective morphisms. See [3, IV, 7.8.4].

\section{REFERENCES}

[1] Atiyah, M. and Mac Donald, I., Introduction to Commutative Algebra, AddisonWesley, Reading, 1969.

[ 2 ] Greco, S., Two theorems on excellent rings, Nagoya Math. J., 60 (1976) 139-149.

[ 3 ] Grothendieck, A. and Dieudonné, J., Eléménts de Géométrie Algébrique, Publ. I.H.E.S., chapp. I, II, IV, 1961, $\cdots$.

[ 4 ] Hartshorne, R., Algebraic Geometry, Springer Verlag, 1977.

[5] Hironaka, H., Resolution of singularities of an algebraic variety over a field of characteristic 0, Ann. of Math., 79 (1964), 109-326.

[6] Matsumura, H., Commutative Algebra, Benjamin, New York, 1972.

[ 7 ] Valabrega, P., P-morfismi e prolungamento di fasci, Rend. Sem. Mat. Univers. Politecn. Torino, 36 (1977-78), 1-18.

Istituto Matematico Università di Siena

Italy 